\title{
Age-Related Macular Degeneration Eyes Presenting with Cuticular Drusen and Reticular Pseudodrusen
}

\author{
Je Moon Yoon \\ Samsung Medical Center, Sungkyunkwan University School of Medicine \\ Dong Hoon Shin \\ Hangil Eye Hospital Retina Center \\ Mingui Kong \\ Hangil Eye Hospital Retina Center \\ Don-Il Ham ( $\sim$ oculus@naver.com) \\ Samsung Medical Center, Sungkyunkwan University School of Medicine
}

\section{Research Article}

Keywords: Age, macular degeneration eyes, cuticular drusen, reticular pseudodrusen, clinical characteristics, patients

Posted Date: December 28th, 2021

DOI: https://doi.org/10.21203/rs.3.rs-1176439/v1

License: (9) (1) This work is licensed under a Creative Commons Attribution 4.0 International License. Read Full License

Version of Record: A version of this preprint was published at Scientific Reports on April 5th, 2022. See the published version at https://doi.org/10.1038/s41598-022-09608-9. 


\section{Abstract}

This study aimed to describe the clinical characteristics of age-related macular degeneration (AMD) eyes with both cuticular drusen (CD) and reticular pseudodrusen (RPD). The clinical records of 13 eyes of seven patients diagnosed with CD and RPD were retrospectively reviewed. All patients underwent a complete ocular examination and multimodal imaging. The distribution patterns of CD (macular and diffuse type) and RPD (localized, intermediate, and diffuse type), presence of soft drusen, large drusen ( $>200 \mu \mathrm{m})$, variant subretinal drusenoid deposits, and macular complications were investigated. The mean age at initial presentation was $71.4 \pm$ 8.8 years and six patients were female. The mean subfoveal choroidal thickness was $143.8 \pm 25.1 \mu \mathrm{m}$. The distribution of CD was of the macular type in all eyes. Distribution of RPD was localized in 11 eyes (84.6\%) and intermediate in two eyes (15.4\%). Soft drusen, large drusen, and variant subretinal drusenoid deposits were present in 13 (100\%), 12 (92.3\%) and, seven (53.8\%) eyes, respectively. Macular neovascularization was observed in two eyes (15.4\%). CD and RPD can coexist in eyes with AMD. Multimodal imaging should be used for AMD eyes with features suggestive of $C D$ and RPD, considering the high likelihood of developing late AMD.

\section{Background}

Age-related macular degeneration (AMD) is the most common cause of blindness in older individuals in industrialized countries. Late AMD, including geographic atrophy (GA) and macular neovascularization (MNV), is the final presentation of irreversible blindness ${ }^{1}$.

Drusen are focal deposits of extracellular debris and are a hallmark of AMD $^{1}$. Many studies, using new imaging methods and histological examinations, have provided further information on drusen. It has been recognized that there are diverse phenotypes of drusen or drusenoid deposits in AMD eyes, including soft drusen, cuticular drusen (CD), and reticular pseudodrusen (RPD) $)^{2,3}$. Interestingly, eyes with CD or RPD showed many imaging and clinical features that differed from those of eyes with only soft drusen.

CD are multiple, small, round drusen that appear hyperfluorescent on fluorescein angiography (FA), showing a "stars-in-the-sky" appearance ${ }^{4}$. CD are widely scattered on the fundus with symmetrical distribution patterns in both eyes. An earlier age of onset and stronger genetic association have been reported for $A M D$ eyes with $C D$, as compared to the rest of the AMD population ${ }^{5}$. CD can cause visual loss as a result of vitelliform macular detachment or late AMD, and could be related to membranoproliferative glomerulonephritis type II and other renal diseases ${ }^{2}$.

RPD are deposits in the subretinal space between photoreceptors and the retinal pigment epithelium, representing morphological features distinct from those of other subtypes of drusen. They appear as bluish-white dots or net-like patterns in color fundus photography (CFP), and subretinal accumulations on optical coherence tomography $(\mathrm{OCT})^{3,6}$. RPD are risk factors for late AMD, particularly GA and type 3 neovascularization 7,8 .

Although there are many reports on CD and RPD, it remains unclear whether CD and RPD can concurrently develop in AMD eyes. An eye manifesting both types may have an additive risk for the development of late AMD, because each of CD and RPD are associated with late AMD. In the present study, we report AMD eyes representing both CD and RPD, and investigate their clinical characteristics, including multimodal imaging features.

\section{Methods}

The clinical records of patients diagnosed with CD or RPD at the retina clinic of Samsung Medical Center, Seoul, Korea, between January 2010 and December 2020, were retrospectively reviewed. Eyes that met the diagnostic criteria for both CD and RPD were included. Exclusion criteria included traumatic, inflammatory, and hereditary retinal disorders.

Demographic information, including age, sex, ocular and systemic comorbidities, and medical history, were obtained for each patient. All patients underwent a comprehensive ophthalmic examination, including measurement of best-corrected visual acuity (BCVA), refractive error by manifest refraction, slit-lamp biomicroscopy, and fundus examination.

Institutional review board approval was obtained from the Samsung Medical Center institutional review board, and the study was performed in accordance with the principles of the Declaration of Helsinki. Given the retrospective nature of the study and the use of anonymized data, the requirement for informed consent was waived by the institutional review board. 
Imaging analysis. Patients underwent multimodal imaging, including CFP (TRC 50 IX, Topcon, Tokyo, Japan), red-free photography $(\mathrm{RF})$, near-infrared reflectance (NIR), fundus autofluorescence (FAF), spectral domain-optical coherence tomography (Spectralis HRA+OCT, Heidelberg Engineering, Heidelberg, Germany), and swept-source optical coherence tomography (DRI OCT Triton, Topcon, Tokyo, Japan). FA, indocyanine green angiography (ICGA; Spectralis HRA+OCT or Optos 200Tx, Optos PLC, Dunfermline, UK), and ultrawide-field (UWF) photography (Optos 200Tx) were also performed. Morphological and topographic features of CD and RPD were assessed by two investigators (J.M.Y and D.H.S.), and in case of disagreement, a senior interpreter (D.I.H.) made the final decision.

The presence of soft drusen, large drusen, MNV, GA, drusenoid pigment epithelial detachment (drusenoid PED), and acquired vitelliform lesion (AVL) was determined as follows. Soft drusen are yellowish white, elevated deposits (>63 $\mu \mathrm{m}$ in size) with slightly blurred boundary in CFP, showing none to minimal hyperfluorescence in later stages of FA. They appear as dome-shaped retinal pigment epithelium (RPE) elevations with homogenous, moderately reflective internal material in OCT. Large drusen were defined as drusen greater than $200 \mu \mathrm{m}$, showing RPE elevations in $\mathrm{OCT}^{19}$. GA was defined as a sharply demarcated hypopigmented area with visible large choroidal vessels in CFP and hypoautofluorescent in FAF, with a diameter of at least $175 \mu \mathrm{m}$. Drusenoid PED was defined as $a \geq 1 / 2$-disc diameter of confluent soft drusen under the center of the macula ${ }^{27}$. VL was defined as a yellowish subretinal material in color photography, and hyperautofluorescence in FAF, corresponding to dome-shaped hyperreflective material between the ellipsoid zone and the RPE/basal lamina/Bruch's membrane band on $\mathrm{OCT}^{28}$.

AMD was classified, according to the Clinical Classification System by the Beckman Initiative for Macular Research Classification Committee. The definition and classification of MNV followed the criteria proposed by the CONAN (Concensus on Neovascular Agerelated macular degeneration Nomenclature) study group.

Diagnosis and distribution of cuticular drusen. The diagnostic criteria for CD used in this study are described in detail elsewhere ${ }^{10}$. In brief, CD were defined as multiple, yellow or pale, small, round lesions observed in CFP, showing a symmetric distribution pattern between bilateral eyes. There had to be at least 50 scattered, uniformly sized, small $(25-75 \mu \mathrm{m})$ hyperfluorescent drusen with a typical "stars-in-the-sky" appearance on FA images in each eye ${ }^{2,5}$. The lesion had to be located beneath the RPE, with RPE elevation on OCT images 2,29 .

Fundus topographic distribution patterns of CD were classified as being either the macular or diffuse type, based on CFP and FA results. The macular type was defined as drusen distributed only within the major vascular arcades, whereas the diffuse type was defined as drusen involving the macula, but also extending beyond the vascular arcades ${ }^{2}$.

Diagnosis and distribution of reticular pseudodrusen. Diagnosis of RPD was based on appropriate findings: 1) multiple yellowish white lesions with a reticular network in CFP, 2) interlacing network in RF imaging, 3) hyporeflectant lesions with mild background hyperreflectance in NIR imaging, 4) hypofluorescent lesions against a background of mild hyperfluorescence in FAF imaging, 5) $\geq 5$ hyperreflective subretinal deposits above the RPE on more than one b-scan image in OCT, and 6) hypofluorescent lesions in the mid- or late-phase of ICGA. RPDs were defined as definite if they were identified using at least three imaging methods, including OCT. The fundus topographic distribution pattern of RPD was classified as localized, intermediate, or diffuse types by the extent of involvement of retinal areas according to criteria described elsewhere ${ }^{23}$. In addition, variant subretinal drusenoid deposits were defined as deposits with features similar to those of RPD, except for hyperautofluorescence on FAF imaging ${ }^{22}$.

\section{Results}

Of the 220 eyes of 110 patients diagnosed with CD and 926 eyes of 463 patients diagnosed with RPD, 13 eyes (5.9\% in CD eyes and $1.4 \%$ in RPD eyes) of seven patients were diagnosed with both CD and RPD (Table 1). Only one eye was included in one patient (Case 4), because the other eye did not meet the diagnostic criteria for RPD. Two patients had systemic hypertension, two had diabetes mellitus, and one had hypothyroidism. There was no case of membranoproliferative glomerulonephritis, which were previously described to be associated with $\mathrm{CD}^{6}$. However, one patient had chronic kidney disease of unknown origin. The mean age was $71.4 \pm$ 8.8 years (range, $57-80$ years), and six patients were female (85.7\%). The mean spherical equivalent was $0.13 \pm 0.43$ diopters (range, -0.75 to +0.88 diopters), and BCVA at first visit was $0.2 \pm 0.4 \operatorname{logMAR}$ (logarithm of the minimum angle of resolution) (range, $0.0-1.3$ logMAR). The average subfoveal choroidal thickness (SFCT) was 143.8 $\pm 25.1 \mu \mathrm{m}$ (range, 103-181 $\mu \mathrm{m}$ ). Eight eyes were pseudophakic, and five eyes were phakic. Two eyes had mild non-proliferative diabetic retinopathy, and two eyes had normal tension glaucoma. 
Multimodal imaging characteristics. CFP, FAF, NIR, OCT, UWF photography, FA, and ICGA were performed in all eyes. RF imaging was performed in nine (69.2\%) eyes.All eyes showed characteristic imaging features of both CD and RPD. Representative cases are shown in Figs. 1-3. In CFP, numerous yellowish and whitish deposits of various sizes were present against a background of pigment disturbance (hypopigmentation and hyperpigmentation). There were larger deposits in the central macular area than in the perimacular area, and small deposits were denser in the superior area than in the inferior area to the macular center. CD appeared as clusters of small, yellow spots, and RPD appeared as slightly bluish-white dots or interlacing networks. However, it was often difficult to distinguish CD from RPD where they coexisted, such as at the superior area of the posterior pole (Fig. 1a) on CFP images. In RF imaging, CD appeared as multiple small, whitish dots in all eyes, but they were less numerous than on FA. RPD appeared as slightly white lesions but were less bright than CD on RF imaging. In NIR imaging, CD showed heterogeneous reflectivity, whereas RPD showed discrete hyporeflectivity with target configurations. In FAF imaging, CD appeared as punctate hypoautofluorescent lesions with a ring of hyperautofluorescence in eight eyes (61.5\%). RPD appeared as hypoautofluorescent lesions in all study eyes, and variant hyperautofluorescent subretinal drusenoid deposits were observed in seven eyes (53.8\%).

The morphological features of CD seen on OCT B-scans can be broadly categorized into two patterns. Three eyes (23.1\%) showed shallow elevations of the RPE-basal laminar band, with druse internal contents being difficult to discern. Ten eyes (76.9\%) showed drusen with triangular morphology, giving a sawtooth appearance, and hyporeflective internal contents. The morphological features of RPD were subretinal deposits with a conical appearance, which occasionally broke through the ellipsoid zone.

FA showed numerous tiny hyperfluorescent spots, akin to a "stars-in-the-sky" appearance, for CD not for RPD. Discrete hyperfluorescence typically occurred in the early arteriovenous frames and persisted throughout the late frames of the angiogram. $C D$ appeared smaller and less numerous on ICGA than on FA. In ICGA, RPD appeared hypofluorescent in all eyes.

The topographic distribution of CD was of the macular type in all eyes. The topographic distribution of RPD was of the localized, intermediate, and diffuse type in $84.6 \%(11 / 13), 15.4 \%(2 / 13)$, and $0 \%(0 / 13)$ of the eyes, respectively.

By analyzing multimodal imaging, soft drusen and large drusen were present in 13 eyes (100\%) and 12 eyes (92.3\%) of the 7 patients, respectively.

Two eyes (15.4\%) had a type $1 \mathrm{MNV}$ and underwent intravitreal injection of anti-vascular endothelial growth factor one year ago (Fig. 4). There were no cases associated with GA, drusenoid PED, or AVL.

Multimodal imaging results of Case 4, 6, and 7 are shown in the Supplementary Figs. S1-3).

\section{Discussion}

The current study showed that CD and RPD coexist in some eyes with AMD. Although Sakurada et al. published photographs of CD presenting along with RPD, there were no reports on case series of AMD eyes with both CD and RPD in a literature search in PubMed ${ }^{9}$. It appears that the development of both $C D$ and RPD in AMD eyes is relatively rare. Only seven cases were found in more than $500 \mathrm{CD}$ or RPD patients.

Patients simultaneously presenting both CD and RPD had demographic features similar to those of patients with CD or RPD. The mean age of the seven patients with CD and RPD was $71.4 \pm 8.8$ years. Compared with previous reports of a Korean population, the mean age of this cohort was older than patients with $\operatorname{CD}(66.6 \pm 9.1$ years $)$ and younger than patients with RPD $(72.6 \pm 9.0 \text { years })^{10,11}$. This cohort showed a female preponderance (85.7\%), similar to Korean patients with CD (82.7\%) or with RPD $(86.2 \%)^{10,11}$.

Although eyes with CD and RPD showed imaging features similar to those of CD or RPD, several features are worth mentioning. First, the mean SFCT of eyes with both CD and RPD was thinner than the previously reported mean SFCT of eyes with CD and eyes with RPD. It was reported that the mean SFCT was $220.1-317.5 \mu \mathrm{m}$ in eyes with CD, and $174.6-186.2 \mu \mathrm{m}$ in eyes with RPD ${ }^{2,10,12-16}$. In the present study, the mean SFCT of eyes with both CD and RPD was $143.8 \pm 25.1 \mu \mathrm{m}$, which was the thinnest among the three groups. Moreover, it was reported that RPD eyes with neovascular AMD had thinner SFCT (145 $\pm 48 \mu \mathrm{m})$ than RPD eyes with dry AMD $(201 \pm 88 \mu \mathrm{m})^{17}$. Eyes with both CD and RPD appear to have very thin SFCT like RPD eyes with neovascular AMD, although only two from 13 eyes had neovascular AMD. A thin choroid is a characteristic feature of RPD, whereas CD is not related to choroidal thinning ${ }^{18}$. Therefore, the choroid of eyes with both RPD and CD appears to be more severely affected by the degeneration process 
than that of RPD eyes. Second, Sakurada et al recently showed that the proportion of CD patients associated with large drusen ( $>200$ $\mu \mathrm{m}$ ) was $34.2 \%^{19}$. In a study of Korean patients, although the definition of large drusen was slightly different (at least three times the greatest height or diameter of typical CD), $59.3 \%$ of eyes with CD had large drusen ${ }^{10}$. In this study, large drusen $(>200 \mu m)$ were accompanied CD and RPD in the same eyes at a much higher rate (92.3\%). Third, none of the eyes in the present study had AVLs. In Caucasian patients with CD, $24.2 \%$ of eyes reportedly develop vitelliform lesions ${ }^{2}$. However, it has been reported that Asian CD patients rarely have AVLs: only $1.2 \%$ of eyes in Korean patients and none in Japanese patients ${ }^{10,20}$. In addition, it has been reported that AVL can be accompanied by RPD ${ }^{21}$. Thus, this small case series also suggests a low prevalence of AVL in Asian patients, even in eyes with both CD and RPD. Fourth, the prevalence of variant subretinal drusenoid deposits was higher in eyes with both CD and RPD $(53.8 \%)$ than in eyes with RPD. It was previously reported that they were uncommonly observed in RPD eyes $(6.6 \%)^{22}$. This finding suggests that the microenvironment of eyes with RPD and CD may differ from that of eyes with RPD only. Fifth, none of the eyes with both CD and RPD had a diffuse-type distribution pattern, in contrast to eyes with CD or RPD. Previous studies on topographic distribution reported that macular and diffuse pattern types were seen in $38.3 \%$ and $61.7 \%$ of eyes with CD, and localized, intermediate, and diffuse types were seen in $30.9 \%, 40.3 \%$, and $28.8 \%$ of eyes with $\operatorname{RPD}^{10,23}$. In the current study, the topographic distribution of CD was of the macular type in all eyes, and the topographic distribution of RPD was of the localized, intermediate, and diffuse type in $84.6 \%(11 / 13), 15.4 \%(2 / 13)$, and $0 \%(0 / 13)$ of eyes, respectively. We speculate that the cellular and molecular environments, where diffuse (probably severe) forms of CD or RPD can develop, are not adequate for the development of both CD and RPD.

We consider that the concurrent presence of $C D$ and RPD may increase the risk of development of late AMD, based on the following findings. The mean SFCT of eyes with both CD and RPD was thinner than the previously reported mean SFCT of eyes with RPD only, and thin choroidal thickness has been reported to be a significant risk factor for developing late AMD in RPD eyes ${ }^{12}$. Large drusen were also found at a much higher rate $(92.3 \%)$ in eyes with both CD and RPD than in eyes with CD only, and it has been reported that $\mathrm{CD}$ eyes associated with large drusen had a high 5-year incidence of late AMD $(51.6 \%)^{19}$. In addition, there might be an additive effect of individual risk by CD and RPD for the development of late AMD. However, there are some controversies regarding the absence of a diffuse distribution type of RPD, which was a risk factor for the development of late AMD in retrospective studies, and the high accompanying rate of variant subretinal drusenoid deposits, which were suggested as a favorable factor for visual prognosis in a small pilot study ${ }^{12,22,23}$.

The current study also showed that an eye could have soft drusen, including large drusen, and even variant subretinal drusenoid deposits, in addition to CD and RPD. Previous studies have reported that $79.2 \%$ and $6.6 \%$ of RPD eyes had soft drusen and variant subretinal drusenoid deposits, respectively ${ }^{12,22}$. In AMD eyes, both soft drusen and RPD were found in $22.1-52.1 \%{ }^{24,25}$. And, in one study investigating fellow eye of unilateral neovascular AMD, the incidence of soft drusen plus RPD was $13.9 \%{ }^{26}$. The coexistence of CD and RPD, with even soft drusen plus variant subretinal drusenoid deposits, indicates that the development of one type of drusen or drusenoid deposit did not interfere with the development of other types of drusen or drusenoid deposits in AMD eyes. There were overlapping topographic distribution areas, where one type of deposit was intermixed with other types of deposits. However, it remains unclear whether they independently developed or shared some common pathogenetic pathways in their development.

Although little is known about the clinical significance of the coexistence of multiple types of drusen or drusenoid deposits in AMD eyes, a few previous studies have suggested that the coexistence of soft drusen and RPD might be associated with some differences in the prognosis. Zweifel et al reported that both soft drusen and RPD were independently correlated with the late AMD ${ }^{24}$. And, one study reported that RPD eyes with other early AMD lesions (soft drusen) in the same eye showed a higher progression rate to late AMD than did RPD eyes with no such lesions (3 years' follow-up, $18.4 \%$ vs. $5 \%)^{12}$. Thus, we suppose that individual type of drusen or drusenoid deposits coexisting in the same eye may be an independent risk factor for late AMD with some additive effect. Further prospective studies are needed in the future.

The current study had several limitations. The sample size of the cohort was small, and the study was retrospective and crosssectional. Eyes with a small number of CD and RPD (probably an early form of disease) might not have been included in this cohort, because a minimum number of deposits was required to meet the diagnostic criteria. In addition, eyes with CD in which RPD regressed due to neovascular AMD or outer retinal atrophy after regression of RPD might not have been included in this cohort. Thus, further studies are needed to investigate the prevalence and clinical features of this disease. 
In conclusion, both CD and RPD can develop in the same eye with AMD. Eyes with both CD and RPD had a thinner SFCT and large drusen more frequently than eyes with CD only or eyes with RPD only. Considering the individual risk associated with thin SFCT and the presence of large drusen, eyes with both $C D$ and RPD may have a higher risk of developing late AMD. Therefore, detection of this form of AMD using multimodal imaging is required for accurate risk assessment. Further studies are needed to investigate the prevalence, clinical features, and prognosis of eyes with both CD and RPD.

\section{Declarations}

\section{Author contributions}

J.M.Y. and D.I.H. designed the research and wrote the main manuscript. J.M.Y., D.H.S. and M.K. performed the data collection and image analysis and prepared figures. D.I.H. reviewed the design, the results, and the final paper. J.M.Y. contributed to the manuscript as first author and D.I.H. contributed to the manuscript as corresponding author. All authors read and approved the final manuscript.

\section{Funding}

The study received no funding

\section{Competing interests}

The authors declare no competing interests.

\section{References}

1. Lim, L. S., Mitchell, P., Seddon, J. M., Holz, F. G. \& Wong, T. Y. Age-related macular degeneration. Lancet 379, 1728-1738. http://doi.org/10.1016/S0140-6736(12)60282-7 (2012).

2. Balaratnasingam, C. et al. Cuticular Drusen: Clinical Phenotypes and Natural History Defined Using Multimodal Imaging. Ophthalmology 125, 100-118. http://doi.org/10.1016/j.ophtha.2017.08.033 (2018).

3. Zweifel, S. A., Spaide, R. F., Curcio, C. A., Malek, G. \& Imamura, Y. Reticular pseudodrusen are subretinal drusenoid deposits. Ophthalmology 117, 303-312 e1. http://doi.org/10.1016/j.ophtha.2009.07.014 (2010).

4. Gass, J. D. M. Stereoscopic Atlas of Macular Diseases: Diagnosis and Treatment. 4th edn 26-30 (Mosby, 1997).

5. van de Ven, J. P. et al. Association analysis of genetic and environmental risk factors in the cuticular drusen subtype of agerelated macular degeneration. Mol. Vis. 18, 2271-2278 (2012).

6. Khan, K. N. et al. Differentiating drusen: Drusen and drusen-like appearances associated with ageing, age-related macular degeneration, inherited eye disease and other pathological processes. Prog. Retin. Eye Res. 53, 70-106. http://doi.org/10.1016/j.preteyeres.2016.04.008 (2016).

7. Schmitz-Valckenberg, S. et al. Reticular drusen associated with geographic atrophy in age-related macular degeneration. Invest. Ophthalmol. Vis. Sci. 52, 5009-5015. http://doi.org/10.1167/iovs.11-7235 (2011).

8. Ueda-Arakawa, N. et al. Prevalence and genomic association of reticular pseudodrusen in age-related macular degeneration. Am. J. Ophthalmol. 155, 260-269 e2. http://doi.org/10.1016/j.ajo.2012.08.011 (2013).

9. Sakurada, Y., Parikh, R. \& Yannuzzi, L. A. Cuticular Drusen presenting with Subretinal Drusenoid Deposits (Pseudodrusen). Ophthalmol. Retina 2, 815. http://doi.org/10.1016/j.oret.2018.03.009 (2018).

10. Shin, D. H., Kong, M., Han, G., Han, J. C. \& Ham, D. I. Clinical manifestations of cuticular drusen in Korean patients. Sci. Rep. 10, 11469. http://doi.org/10.1038/s41598-020-68493-2 (2020).

11. Lee, M. Y., Yoon, J. \& Ham, D. I. Clinical characteristics of reticular pseudodrusen in Korean patients. Am. J. Ophthalmol. 153, 530-535. http://doi.org/10.1016/j.ajo.2011.08.012 (2012).

12. Kong, M., Kim, S. \& Ham, D. I. Incidence of Late Age-Related Macular Degeneration in Eyes with Reticular Pseudodrusen. Retina 39, 1945-1952. http://doi.org/10.1097/IAE.0000000000002263 (2019).

13. Mrejen-Uretsky, S., Ayrault, S., Nghiem-Buffet, S., Quentel, G. \& Cohen, S. Y. Choroidal Thickening in Patients with Cuticular Drusen Combined with Vitelliform Macular Detachment. Retina 36, 1111-1118. http://doi.org/10.1097/IAE.0000000000000831 (2016). 
14. Kumawat, D., Padhy, S. K. \& Kumar, V. Clinical and Multimodal Imaging Features of Subretinal Drusenoid Deposits. J. Ophthalmic. Vis. Res. 16, 187-194. http://doi.org/10.18502/jovr.v16i2.9082 (2021).

15. Querques, G. et al. Choroidal changes associated with reticular pseudodrusen. Invest. Ophthalmol. Vis. Sci. 53, 1258-1263. http://doi.org/10.1167/iovs.11-8907 (2012).

16. Mrejen, S. \& Spaide, R. F. The relationship between pseudodrusen and choroidal thickness. Retina 34, 1560-1566. http://doi.org/10.1097/IAE.0000000000000139 (2014).

17. Haas, P., Esmaeelpour, M., Ansari-Shahrezaei, S., Drexler, W. \& Binder, S. Choroidal thickness in patients with reticular pseudodrusen using 3D 1060-nm OCT maps. Invest. Ophthalmol. Vis. Sci. 55, 2674-2681. http://doi.org/10.1167/iovs.13-13338 (2014).

18. Switzer, D. W., Jr., Mendonca, L. S., Saito, M., Zweifel, S. A. \& Spaide, R. F. Segregation of ophthalmoscopic characteristics according to choroidal thickness in patients with early age-related macular degeneration. Retina 32, 1265-1271. http://doi.org/10.1097/IAE.0b013e31824453ac (2012).

19. Sakurada, Y. et al. CUTICULAR DRUSEN: Risk of Geographic Atrophy and Macular Neovascularization. Retina 40, $257-265$. http://doi.org/10.1097/IAE.0000000000002399 (2020).

20. Sakurada, Y. et al. Clinical characteristics of cuticular drusen in the Japanese population. Jpn. J. Ophthalmol. 63, 448-456. http://doi.org/10.1007/s10384-019-00692-5 (2019).

21. Zweifel, S. A., Spaide, R. F. \& Yannuzzi, L. A. Acquired vitelliform detachment in patients with subretinal drusenoid deposits (reticular pseudodrusen). Retina 31, 229-234. http://doi.org/10.1097/IAE.0b013e3181f049bd (2011).

22. Lee, M. Y. \& Ham, D. I. Subretinal drusenoid deposits with increased autofluorescence in eyes with reticular pseudodrusen. Retina 34, 69-76. http://doi.org/10.1097/IAE.0b013e318295f701 (2014).

23. Lee, M. Y., Yoon, J. \& Ham, D. I. Clinical features of reticular pseudodrusen according to the fundus distribution. Br. J. Ophthalmol. 96, 1222-1226. http://doi.org/10.1136/bjophthalmol-2011-301207 (2012).

24. Zweifel, S. A., Imamura, Y., Spaide, T. C., Fujiwara, T. \& Spaide, R. F. Prevalence and significance of subretinal drusenoid deposits (reticular pseudodrusen) in age-related macular degeneration. Ophthalmology 117, 1775-1781. http://doi.org/10.1016/j.ophtha.2010.01.027 (2010).

25. Zarubina, A. V. et al. Prevalence of Subretinal Drusenoid Deposits in Older Persons with and without Age-Related Macular Degeneration, by Multimodal Imaging. Ophthalmology 123, 1090-1100. http://doi.org/10.1016/j.ophtha.2015.12.034 (2016).

26. Lee, J. et al. Neovascularization in Fellow Eye of Unilateral Neovascular Age-related Macular Degeneration According to Different Drusen Types. Am. J. Ophthalmol. 208, 103-110. http://doi.org/10.1016/j.ajo.2019.07.013 (2019).

27. Roquet, W., Roudot-Thoraval, F., Coscas, G. \& Soubrane, G. Clinical features of drusenoid pigment epithelial detachment in age related macular degeneration. Br. J. Ophthalmol. 88, 638-642. http://doi.org/10.1136/bjo.2003.017632 (2004).

28. Freund, K. B. et al. Acquired Vitelliform Lesions: correlation of clinical findings and multiple imaging analyses. Retina 31, 13-25. http://doi.org/10.1097/IAE.0b013e3181ea48ba (2011).

29. Leng, T., Rosenfeld, P. J., Gregori, G., Puliafito, C. A. \& Punjabi, O. S. Spectral domain optical coherence tomography characteristics of cuticular drusen. Retina 29, 988-993. http://doi.org/10.1097/IAE.0b013e3181ae7113 (2009).

\section{Tables}

Table 1. Characteristics of eyes presenting with cuticular drusen and reticular pseudodrusen 


\begin{tabular}{|c|c|c|c|c|c|c|c|c|c|c|c|c|}
\hline & Age (years) & Sex & Laterality & $\begin{array}{l}\text { BCVA } \\
\text { (logMAR) }\end{array}$ & $\begin{array}{r}\text { SFCT } \\
(\mu \mathrm{m})\end{array}$ & $\begin{array}{l}\text { Distribution } \\
\text { of } C D\end{array}$ & $\begin{array}{l}\text { Distribution } \\
\text { of RPD }\end{array}$ & $\begin{array}{l}\text { Soft } \\
\text { drusen }\end{array}$ & $\begin{array}{l}\text { Large } \\
\text { drusen } \\
(> \\
200 \\
\mu \mathrm{m})\end{array}$ & $\begin{array}{l}\text { Variant } \\
\text { SDD }\end{array}$ & $\begin{array}{l}\text { Macular } \\
\text { complications }\end{array}$ & $\begin{array}{l}\text { Ocular } \\
\text { comorbidities }\end{array}$ \\
\hline \multirow[t]{2}{*}{$\begin{array}{l}\text { Case } \\
1\end{array}$} & 73 & $\mathrm{~F}$ & OD & 0.2 & 138 & Macula & Localized & + & + & - & & \\
\hline & & & OS & 1.0 & 176 & Macula & Localized & + & + & - & & \\
\hline \multirow[t]{2}{*}{$\begin{array}{l}\text { Case } \\
2 \\
\end{array}$} & 75 & $\mathrm{~F}$ & OD & 0.0 & 131 & Macula & Localized & + & + & + & & \\
\hline & & & OS & 0.0 & 138 & Macula & Localized & + & + & + & & \\
\hline \multirow[t]{2}{*}{$\begin{array}{l}\text { Case } \\
3 \\
\end{array}$} & 62 & $\mathrm{~F}$ & OD & 0.1 & 110 & Macula & Intermediate & + & + & + & & \\
\hline & & & OS & 0.1 & 103 & Macula & Intermediate & + & + & + & & \\
\hline $\begin{array}{l}\text { Case } \\
4\end{array}$ & 57 & $\mathrm{M}$ & OS & 0.0 & 143 & Macula & Localized & + & - & - & & \\
\hline \multirow[t]{2}{*}{$\begin{array}{l}\text { Case } \\
5\end{array}$} & 80 & $\mathrm{~F}$ & OD & 1.3 & 141 & Macula & Localized & + & + & - & MNV type 1 & NPDR \\
\hline & & & OS & 0.1 & 176 & Macula & Localized & + & + & + & MNV type 1 & NPDR \\
\hline \multirow[t]{2}{*}{$\begin{array}{l}\text { Case } \\
6 \\
\end{array}$} & 73 & $\mathrm{~F}$ & OD & 0.0 & 181 & Macula & Localized & + & + & + & & \\
\hline & & & OS & 0.0 & 171 & Macula & Localized & + & + & + & & \\
\hline \multirow[t]{2}{*}{$\begin{array}{l}\text { Case } \\
7 \\
\end{array}$} & 80 & $\mathrm{~F}$ & OD & 0.0 & 134 & Macula & Localized & + & + & - & & NTG \\
\hline & & & OS & 0.1 & 128 & Macula & Localized & + & + & - & & NTG \\
\hline
\end{tabular}

BCVA, best-corrected visual acuity; CD, cuticular drusen; logMAR, logarithm of the minimum angle of resolution; MNV, macular neovascularization; NPDR, non-proliferative diabetic retinopathy; NTG, normal tension glaucoma; RPD, reticular pseudodrusen; SDD, subretinal drusenoid deposit; SFCT, subfoveal choroidal thickness; M, male; F, female; OD, right eye; OS, left eye.

\section{Figures}



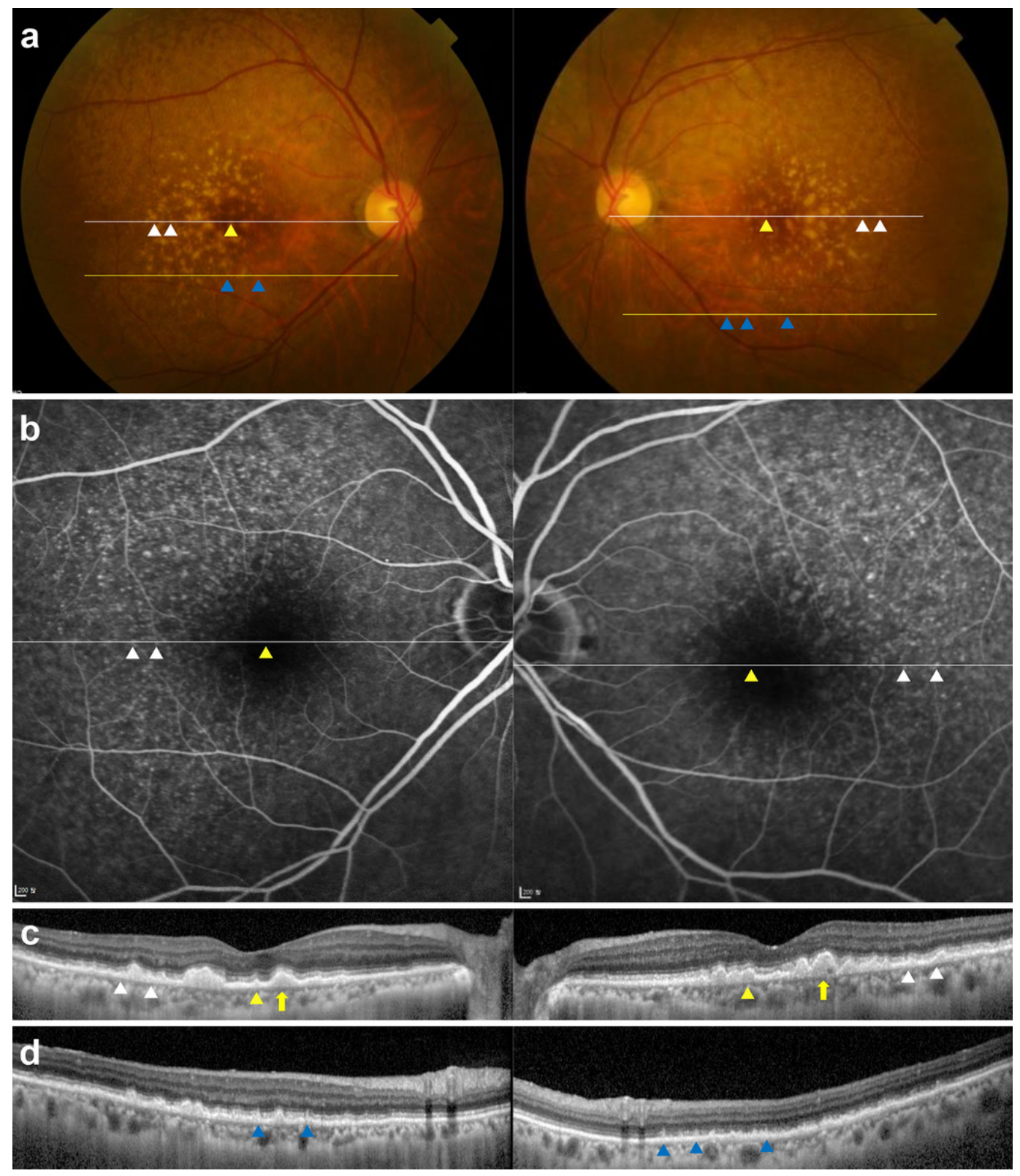

\section{Figure 1}

Multimodal Imagings of a 73-year-old woman (Case 1). (a) Numerous yellowish and whitish deposits of various sizes are present in color fundus photograph in both eyes. (b) Fluorescein angiography (FA) shows multiple hyperfluorescence of "stars-in-the-sky" appearance. (c) Optical coherence tomography (OCT) scan (white line in A and B) shows sub-retinal pigment epithelium (RPE) deposits. Some of them shows hyperfluorescence in FA (cuticular drusen, white arrowhead), and others shows hypofluorescence in FA (soft drusen, yellow arrowhead). Large drusen $(>200 \mu \mathrm{m}$ ) is also observed (yellow arrow). (d) OCT scan, obtained at the location of the yellow line in $A$, shows subretinal deposits with a conical appearance, which break through the ellipsoid zone, corresponding to reticular pseudodrusen (blue arrowhead). They have no hyperfluorescence in FA. 

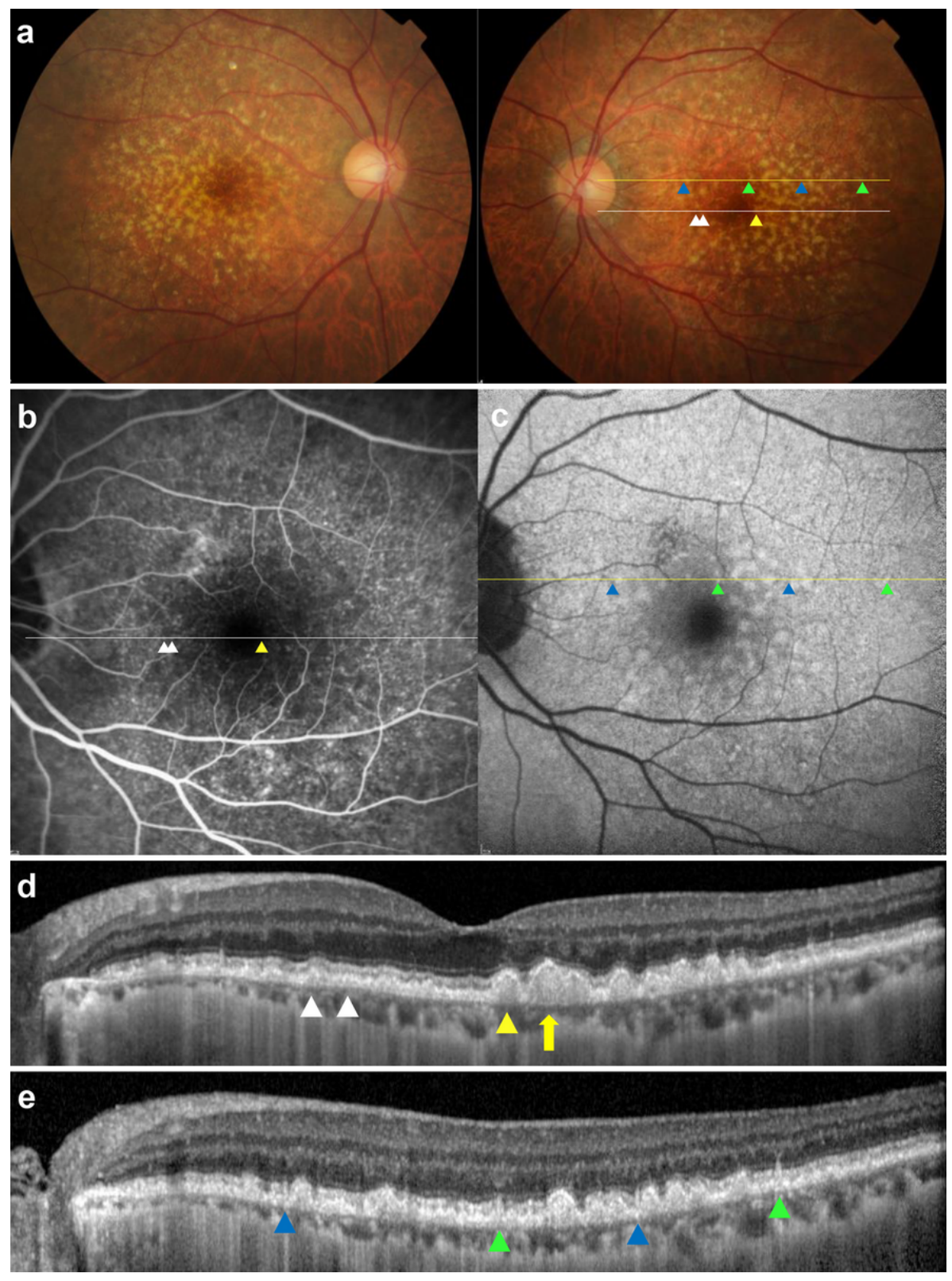

\section{Figure 2}

Multimodal Imagings of a 75-year-old woman (Case 2). (a) Color fundus photograph shows small to large deposits at the posterior pole in both eyes. (b) Fluorescein angiography (FA) shows numerous hyperfluorescent spots, and (c) fundus autofluorescence shows numerous hypoaurofluorescent and hyperautofluorescent spots. (d) Optical coherence tomography (OCT) scan (white line in A and B) shows saw-tooth elevation of retinal pigment epithelium (RPE) at the position of hyperfluorescent spots in FA, corresponding to culticular drusen (white arrowhead). Yellow arrowhead indicates sub-RPE deposit with no hyperfluorescence in FA (soft drusen). Large drusen $(>200 \mu \mathrm{m}$ ) is also observed (yellow arrow). (e) OCT scan at the location of the yellow line in A and C shows subretinal deposits, corresponding to reticular pseudodrusen (blue arrowhead) and variant subretinal drusenoid deposits (green arrowhead). 

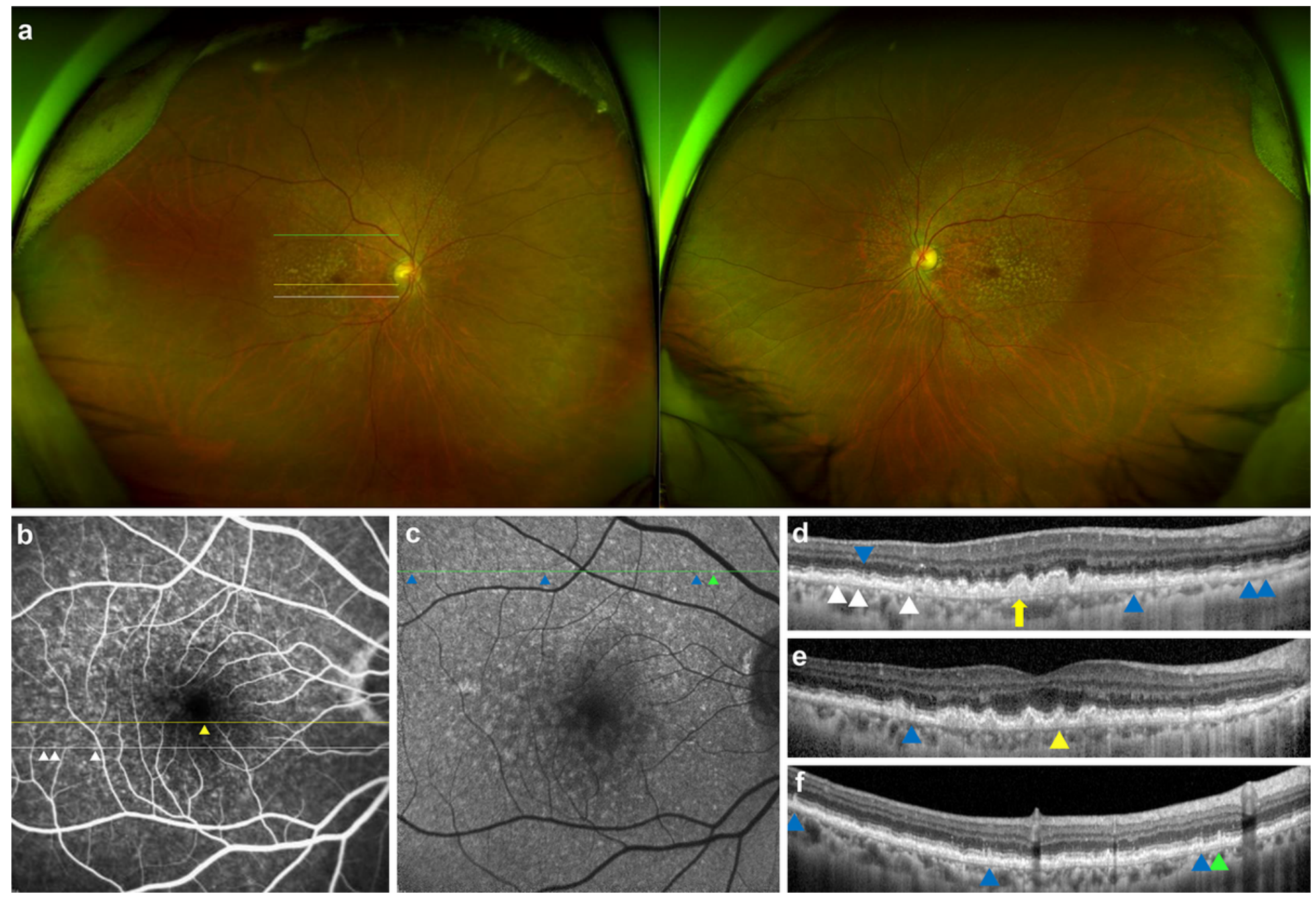

Figure 3

Multimodal imagings of a 62-year-old woman (Case 3). (a) Numerous yellowish and whitish deposits of various sizes are present in ultrawide-field photograph, and whitish deposits (reticular pseudodrusen) exist beyond the major arcades in both eyes. (b) Fluorescein angiography shows multiple hyperfluorescence of "stars-in-the-sky" appearance within the major vascular arcades. (c) Fundus autofluorescence shows numerous hypoaurofluorescent and hyperautofluorescent spots. (d) Optical coherence tomography (OCT) scan (white line in A and B) shows saw-tooth elevation of retinal pigment epithelium (RPE) corresponding to cuticular drusen (white arrowhead). Multiple subretinal deposits, corresponding to reticular pseudodrusen (blue arrowhead) and large drusen (yellow arrow, > $200 \mu \mathrm{m}$ ) are also observed. (e) OCT scan taken at the position of the yellow line in A and B shows sub-RPE deposit (yellow arrowhead) showing no fluorescence on Image B (soft drusen). A blue arrowhead indicates a reticular pseudodrusen. (f) OCT scan, obtained at the location of the green line in $\mathrm{A}$ and $\mathrm{C}$, shows subretinal deposits corresponding to reticular pseudodrusen (blue arrowhead) and variant subretinal drusenoid deposits (green arrowhead). 

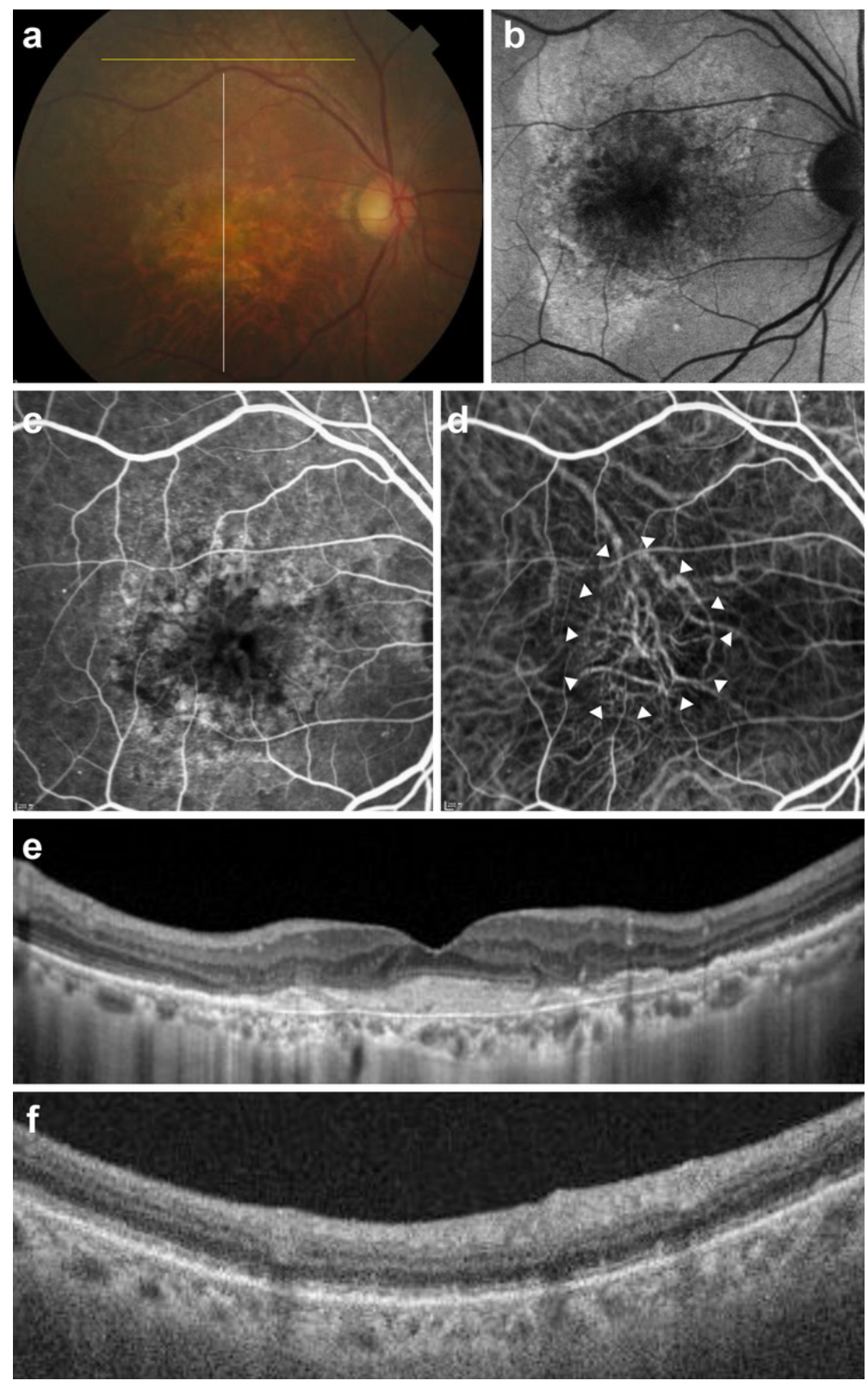

\section{Figure 4}

Multimodal imagings of an 80-year-old woman who had macular neovascularization and non-proliferative diabetic retinopathy (Case 5). (a) Multiple deposits of various sizes are present at the macula and the area superior to the macula in color fundus photography. Macular hypopigmentation is also observed. (b) Fluorescein angiography shows perifoveal hyperfluorescent areas, microaneurysms, and small hyperfluorescent spots corresponding to cuticular drusen. (c) Indocyanine green angiography (ICGA) shows neovascularization (white arrowheads). (d) Optical coherence tomography (OCT) scan, obtained at the location of the white line in A, shows saw-tooth elevation of retinal pigment epithelium, subretinal hyperreflective material, and pigment epithelial detachment. (e) OCT scan, obtained at the position of the yellow line, shows subretinal deposits corresponding to reticular pseudodrusen.

\section{Supplementary Files}

This is a list of supplementary files associated with this preprint. Click to download.

- SFigure1.tif

- SFigure2.tif

- SFigure3.tif 